\title{
Une année de rêve, une année de science
}

I faut se rendre à l'évidence, il existe des années plus égales que les autres et, à ce palmarès, l'année 2000 l'emporte haut la main sur toutes celles que notre génération a vécues. Quels que soient les succès que l'humanité remportera cette année, et l'on ne voit pas a priori pourquoi il y en aurait moins que d'habitude mais pas non plus pourquoi il y en aurait plus ou de plus décisifs, l'année 2000 sera exceptionnelle par la force de rêve qu'elle porte inévitablement en elle. Comme si sa place à l'entrée du millénaire faisait de cette année une fenêtre par laquelle les hommes de notre temps ne pouvaient s'empêcher de regarder l'avenir. Rêver, les scientifiques sont certainement parmi ceux pour lesquels ce terme correspond le mieux à une activité quotidienne, à un véritable mode de fonctionnement. L'année 2000, année de rêve, sera donc par essence l'année de la science, l'année des scientifiques, constructeurs d'avenir, metteurs en scène de rêves. Elle sera même, très largement, l'année des sciences de la vie tant il paraît évident que, au milieu de tous les succès scientifiques de notre période, l'identification des milliards de bases qui forment le génome de notre espèce demeure un événement extraordinaire qui marquera pour les générations futures l'ouverture (même si sa réalisation finale n'interviendra que dans deux ou trois ans) de ce IIIe millénaire.

médecine/sciences ne pouvait donc que se lancer, elle aussi, dans la célébration de cette année au nombre symbolique. Nous avons décidé de le faire en offrant à nos lecteurs tout au long de l'année encore plus de science, et encore plus de rêve, au travers d'une série de numéros exceptionnels dont les sommaires ont été élaborés spécialement par les membres des comités de médecine/ sciences. Dans chacun des dix numéros de cette année médecine/sciences contiendra ainsi, en plus de ses rubriques habituelles, un ensemble d'articles regroupés dans ce que nous avons appelé les «cahiers 2000», qui tenteront sinon de faire le tour d'un des grands thèmes de la recherche biomédicale, cela serait une gageure intenable, du moins d'en évoquer des aspects essentiels et porteurs d'avenir. De la «révolution du génome» qui ouvrira naturellement cette série, jusqu'aux «grandes orientations théra- peutiques» qui la concluront en décembre prochain comme pour mieux montrer l'objectif premier de nos recherches, les 10 «cahiers 2000»de médecine/sciences (dont on trouvera les titres sur la page suivante) présenteront, au-delà des bilans et des projets, des visions. Ces visions de leur domaine de recherche, de leurs réalisations et de leur devenir, nous les avons demandées à une centaine de scientifiques parmi les plus prestigieux de notre communauté francophone. Tout au long de l'année, ces chercheurs présenteront ainsi aux lecteurs de médecine/sciences les résultats scientifiques qui fondent la connaissance sur laquelle vont s'appuyer bien des travaux de demain, mais encore et peutêtre surtout la marche de la recherche biomédicale, ses interrogations et ses espoirs. Et parce que les artistes sont, avec les scientifiques, les rêveurs les plus accomplis de notre temps, nous avons décidé de promouvoir au mieux en cette année exceptionnelle la symbiose entre les deux communautés. Sur proposition d'Hervé Chneiweiss, qui organisera cette rencontre toute l'année, nous avons ainsi invité 10 grands artistes à ouvrir chacun un des numéros de médecine/ sciences de l'an 2000 d'une œuvre originale inspirée par le thème.

Des interrogations et des espoirs, l'année qui vient de s'écouler en a comme ses précédentes apporté son lot. Parmi eux, on ne s'étonnera pas que nous choisissions dans ces vœux de faire une place à part à l'initiative d'Harold Varmus en vue de la création d'E-biomed, le système de communication scientifique instantané et ouvert en sciences de la vie. L'information scientifique est au cœur de toute activité de recherche et, à ce titre, mérite un traitement tout à fait particulier à la hauteur des efforts qui la nourrissent et de ceux auxquels elle contribue. La dispersion actuelle des articles scientifiques dans des revues multiples, chères, pour beaucoup difficiles d'accès, est un des freins les plus évidents au développement de la recherche dans nos domaines, comme elle est une des charges financières les plus lourdes de nos instituts. A l'heure où le monde s'engouffre dans la civilisation de l'information totale au travers des lignes à hauts débits, cette situation de la recherche biomédicale fait figure d'héritage du passé, dépassé. Il y a d'ailleurs bien longtemps que d'autres communautés scientifiques ont franchi le pas et s'en portent fort bien, les physiciens par exemple chez lesquels l'idée qu'un article puisse mettre des semaines, voire des mois à arriver jusqu'à ceux qui en ont besoin paraîtrait de la plus grande incongruité, a fortiori l'idée qu'il faudrait dépenser des sommes considérables pour y avoir accès. La proposition d'Harold Varmus a fait couler beaucoup d'encre, et lever bien des boucliers dont certains défendaient de façon évidente plus un intérêt commercial que celui de la science. Il serait malheureux que l'enjeu scientifique puisse passer au second plan, mais des affaires récentes rendent plutôt pessimiste. Ainsi, durant l'année qui vient de s'écouler, la protestation de la communauté n'a pu empêcher que Jerome Kassirer soit limogé du poste de rédacteur en chef du New England Journal of Medicine, qu'il occupait depuis 8 ans, parce qu'il s'opposait à une stratégie commerciale des propriétaires du journal qu'il estimait contraire au maintien de sa qualité scientifique. Il n'est pas sûr, aujourd'hui, qu'E-biomed existe réellement au début de l'année 2000, au moment où son initiateur quitte la direction des National Institutes of Health. médecine/sciences n'avait pas jusqu'à présent directement participé à ce débat sur l'initiative d'Harold Varmus, son poids ne lui permettant pas vraiment de peser sur le cours des événements. L'existence de notre revue depuis 15 ans est pourtant en elle-même une expression de la volonté de l'ensemble de notre communauté d'une information scientifique plus large et plus accessible. C'est donc absolument sans réserve que nous formulons, puisque c'en est l'époque, le vœu que le rêve d'une communication scientifique immédiate, complète, libre et gratuite, que font tous les chercheurs et qu'Harold Varmus a essayé de concrétiser, devienne au plus vite réalité. $\mathrm{Au}$ nom de tous les membres du bureau éditorial et des comités de médecine/ sciences, nous souhaitons à tous nos lecteurs une année 2000 riche d'enthousiasmes, de découvertes et de réussites... une année de rêve !

Marc Peschanski Rédacteur en chef, Paris Michel Bergeron Rédacteur en chef, Montréal 